\title{
Sensitivity enhanced roll-angle sensor by means of a quarter-waveplate
}

\author{
Empfindlichkeitssteigernder Rollwinkelsensor mittels einer Viertelwellenplatte
}

\section{DOI 10.1515/teme-2021-0069}

\begin{abstract}
Attitude metrology (roll, pitch, and yaw) plays an important role in many different fields. Roll angle is considered the most difficult measurement quantity in angular displacements compared to pitch and yaw angles because the rotation axis of the roll angle is parallel to the probe beam. In this work, a sensitivity enhanced roll-angle sensor is presented. The principle is based on the polarization change of a sensing unit (quarter-waveplate). The polarization model is analyzed by Mueller matrix formalism. The Stokes parameters are detected by a Stokes polarimeter. The novel coaxial design improves the sensitivity and reduce the complexity of optical system alignment by means of a fixed quarter-waveplate. The proposed sensor provides a simple setup to measure roll angles with a high sensitivity of $0.006^{\circ}$ and a long unambiguous measurement range of $180^{\circ}$.
\end{abstract}

Keywords: Roll angle, angle sensor, polarization sensor

Zusammenfassung: Winkelmessungen (Rollen, Neigen und Gieren) spielen in vielen verschiedenen Bereichen eine wichtige Rolle. Der Rollwinkel wird als die schwierigste Messgröße bei Winkelverschiebungen im Vergleich zu Nickund Gierwinkeln angesehen, da die Rotationsachse des Rollwinkels parallel zum Sondenstrahl verläuft. In dieser Arbeit wird ein sensitivitätsverbesserter Rollwinkelsensor vorgestellt. Das Prinzip basiert auf der Polarisationsänderung der Sensoreinheit (Viertelwellenplatte). Das Polarisationsmodell wird durch den Müller-Matrixformalismus analysiert. Die Stokes-Vektoren werden von einem StokesPolarimeter erfasst. Das neuartige koaxiale Design verbes-

\footnotetext{
*Corresponding author: Chia-Wei Chen, Vision and Fusion Laboratory (IES), Karlsruhe Institute of Technology (KIT), and Fraunhofer Institute of Optronics, System Technologies and Image Exploitation IOSB, E-Mail: chia-wei.chen@kit.edu; chia-wei.chen@iosb.fraunhofer.de Matthias Hartrumpf, Thomas Längle, Fraunhofer Institute of Optronics, System Technologies and Image Exploitation IOSB Jürgen Beyerer, Vision and Fusion Laboratory (IES), Karlsruhe Institute of Technology (KIT), and Fraunhofer Institute of Optronics, System Technologies and Image Exploitation IOSB
}

sert die Empfindlichkeit und reduziert die Komplexität der Systemausrichtung mithilfe einer festen Viertelwellenplatte. Der vorgeschlagene Sensor bietet einen einfachen Aufbau zum Messen von Rollwinkeln mit einer hohen Auflösung von $0.006^{\circ}$ und einem langen eindeutigen Messbereich von $180^{\circ}$.

Schlüsselwörter: Rollwinkel, Winkelsensor, Polarisationssensor

\section{Introduction}

Attitude metrology (roll, pitch, and yaw) plays an important role in many different fields, e.g., precision machinery [2], remote sensing [4] and space docking [17]. Compared to pitch and yaw angles, roll angle is considered the most difficult measurement quantity in angular displacements because the rotation axis of the roll angle is parallel to the probe beam[15]. State-of-the-art technologies for roll-angle measurements are autocollimator [10], optoelectronic level [5], relative position shift of two parallel laser beams [2], rotary encoder [16] and variation of polarization states [14]. Except for the last two methods, the measurement range of other methods is limited to few degrees or few arcminutes [16]. Rotary encoders can only be applied when the measured object has a fixed axis of rotation. In other words, the distance between the optical head and the disk scale is fixed. This constraint limits the feasibility for long-range measurements, e.g., accuracy calibration of precision machines and orientation tracking for robots. In general, the polarization-based measurement can provide a large measuring range with a high resolution and the distance between the sensing unit and the detector can be flexible. Li et al. proposed a compact optical roll-angle sensor in a range of $\pm 30^{\circ}$ with a resolution of $0.01^{\circ}$ by using a Faraday rotator as a sensing unit [12]. Kuang et al. measured the roll angle in a range of $\pm 30^{\circ}$ with a resolution of 0.04 arcminutes [11]. Shi and Guo introduced a new concept for roll-angle measurements by using a half-waveplate and a right angle prism[15]. Gillmer et al. demonstrated a roll sensor in a working range of $43^{\circ}$ with 
a resolution of $0.002^{\circ}$ by measuring the azimuthal angle of a half-waveplate with modulated signals [7]. Chen et al. presented an absolute roll-angle sensor using a retarder as a sensing unit in a range of $180^{\circ}$ with a resolution of $0.01^{\circ}$ [4]. In this paper, we propose a new roll-angle sensor based on the concept of Shi and Guo[15]. We replace the right angle prism with a quarter-waveplate (QWP) and a plain mirror to achieve a coaxial design. The roll angle is obtained by measuring the change of the polarization states of a sensing unit (QWP). The feature of the proposed sensor is the high sensitivity of the roll-angle measurement with a large measurement range.

\section{Measurement principle}

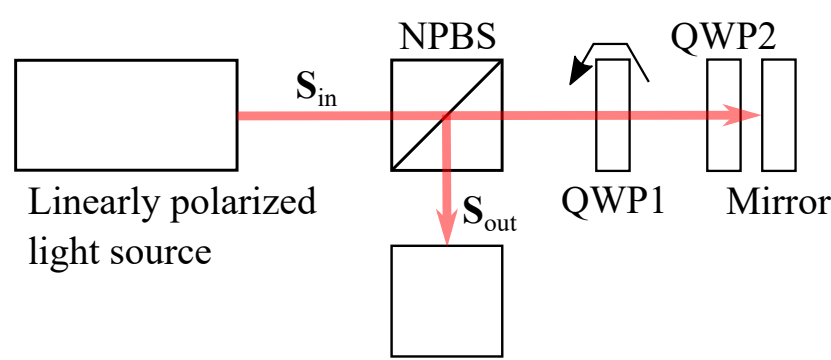

Stokes polarimeter

Fig. 1: The schematic of the roll-angle sensor, where $\mathbf{S}_{\text {in }}$ and $\mathbf{S}_{\text {out }}$ are the Stokes vectors of the linear polarized light source and the return beam reflected by the NPBS.

Figure 1 shows the schematic of the proposed rollangle sensor. The linearly polarized light passed through a non-polarizing beamsplitter (NPBS), two quarterwaveplates (QWP1 and QWP2), and the returned beam was reflected by the NPBS and ultimately received by a Stokes polarimeter. In the system, QWP1 is a sensing unit for the roll angle and the other components are fixed. The polarization effect of optical elements or interaction at boundaries can be described by Stokes vectors and Mueller matrices [6]. Stoke vectors $\mathbf{S}$ describe the polarization state of light beams. Mueller matrices $\mathbf{M}$ represent the characteristics of the altering of Stokes vectors when light interacts with matter.

$$
\mathbf{S}=\left[\begin{array}{l}
s_{0} \\
s_{1} \\
s_{2} \\
s_{3}
\end{array}\right], \mathbf{M}=\left[\begin{array}{llll}
m_{11} & m_{12} & m_{13} & m_{14} \\
m_{21} & m_{22} & m_{23} & m_{24} \\
m_{31} & m_{32} & m_{33} & m_{34} \\
m_{41} & m_{42} & m_{43} & m_{44}
\end{array}\right]
$$

Equation 2 shows the polarization model of the measurement described by Stokes vectors and Mueller matrices, where $\mathbf{M}_{\mathrm{NPBS}}, \mathbf{M}_{\mathrm{QWP}}$ and $\mathbf{M}_{\text {Mirror }}$ are the Muller matrices of the NPBS, the QWP and the mirror, and r, t, $\theta$ and $\delta$ denote the reflection and the transmission of the NPBS and the fast-axis orientation angle and the retardance of the QWP, respectively. The full form of $\mathbf{M}_{\mathrm{QWP}}$ is shown in Eq. 3. If every element is ideal, $\mathbf{M}_{\mathrm{NPBS}}^{\mathrm{r}}$ and $\mathbf{M}_{\text {Mirror }}$ are diagonal matrices, where the diagonal elements are $1,1,-1$, and -1 . $\mathbf{M}_{\mathrm{NPBS}}^{\mathrm{r}}$ is a diagonal matrix with diagonal elements $1,1,1$, and 1 . For simplicity, we set $\delta_{1}=\delta_{2}=90^{\circ}, \theta_{2}=0^{\circ}$ and $\mathbf{S}_{\mathrm{in}}=\left[\begin{array}{llll}1 & 1 & 0 & 0\end{array}\right]^{\mathrm{T}}$. Then, the measurement result can be expressed as:

$$
\mathbf{S}_{\mathrm{out}}=\left[\begin{array}{l}
s_{0} \\
s_{1} \\
s_{2} \\
s_{3}
\end{array}\right]=\left[\begin{array}{c}
1 \\
\frac{1}{4}\left(3+\cos 8 \theta_{1}\right) \\
-\sin ^{2} 2 \theta_{1} \sin 4 \theta_{1} \\
-\cos 2 \theta_{1} \sin 4 \theta_{1}
\end{array}\right]
$$

where $\theta_{1}$ can be seen as the roll angle.

Figure 2 presents simulated Stokes parameters for the roll angle from $0^{\circ}$ to $180^{\circ}$. It is obvious that the periods of $s_{1}, s_{2}, s_{3}$ are $45^{\circ}, 90^{\circ}$ and $180^{\circ}$, separately. The Stokes polarimeter can measure full Stokes parameters. Therefore, the roll angle can be solved by $s_{1}, s_{2}$ or $s_{3}$ in Eq. 4 analytically. For better visualization of the evolution of $\mathbf{S}_{\text {out }}, \mathbf{S}_{\text {out }}$ are mapped to the Poincaré sphere as shown in Fig. 3. Compared to the work from Chen et al. [4], the period of $s_{1}$ is reduced from $90^{\circ}$ to $45^{\circ}$. In theory, the resolution could be enhanced twice. Since $s_{1}$ is a cosine wave, it is feasible to use commercial encoders to interpolate the signal. If $s_{1}$ can be interpolated with a 14-bit encoder, the resolution can be achieved to $0.003^{\circ}$ and the unambiguous range is $180^{\circ}$. Therefore, this configuration has a high sensitivity and a large measurement range for roll-angle measurements.

\section{Experiment setup}

Figure 4 shows the prototype of the roll-angle sensor which consists of a laser with a wavelength of $638 \mathrm{~nm}$ from Integrated Optics, a linear polarizer (PGT 3.05, B. halle Nachfl.GmbH), a NPBS (CM1-BP145B1, Thorlabs, Inc.) two QWPs (WPQ10ME-633, Thorlabs, Inc.), a silver mirror and a Stokes polarimeter (PAX1000VIS, Thorlabs, Inc.). QWP1 is mounted on a piezo rotation stage (M-660.55, Physik Instrumente GmbH). Compared to polarizing beamsplitters, the NPBS has strong polarization distortions $[9,13,18]$. The wavelength mismatch between the laser and the QWPs also induces retardance 


$$
\mathbf{S}_{\mathrm{out}}=\mathbf{M}_{\mathrm{NPBS}}^{\mathrm{r}} \mathbf{M}_{\mathrm{QWP} 1}\left(-\theta_{1}, \delta_{1}\right) \mathbf{M}_{\mathrm{QWP} 2}\left(-\theta_{2}, \delta_{2}\right) \mathbf{M}_{\mathrm{Mirror}} \mathbf{M}_{\mathrm{QWP} 2}\left(\theta_{2}, \delta_{2}\right) \mathbf{M}_{\mathrm{QWP} 1}\left(\theta_{1}, \delta_{1}\right) \mathbf{M}_{\mathrm{NPBS}}^{\mathrm{t}} \mathbf{S}_{\mathrm{in}} .
$$

$$
\mathbf{M}_{\mathrm{QWP}}(\theta, \delta)=\left[\begin{array}{cccc}
1 & 0 & 0 & 0 \\
0 & \cos ^{2} 2 \theta+\sin ^{2} 2 \theta \cos \delta & (1-\cos \delta) \sin 2 \theta \cos 2 \theta & -\sin 2 \theta \sin \delta \\
0 & (1-\cos \delta) \sin 2 \theta \cos 2 \theta & \sin ^{2} 2 \theta+\cos ^{2} 2 \theta \cos \delta & \cos 2 \theta \sin \delta \\
0 & \sin 2 \theta \sin \delta & -\cos 2 \theta \sin \delta & \cos \delta
\end{array}\right]
$$

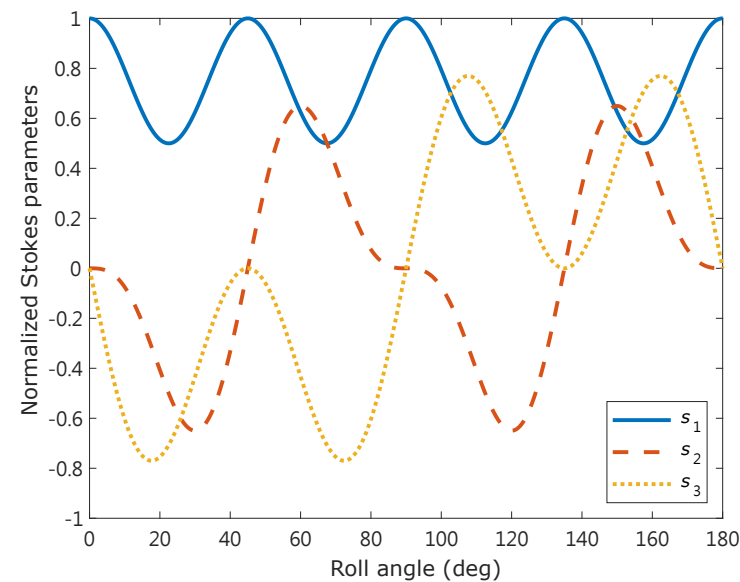

Fig. 2: Simulated Stokes parameters for the roll angle from $0^{\circ}$ to $180^{\circ}$.

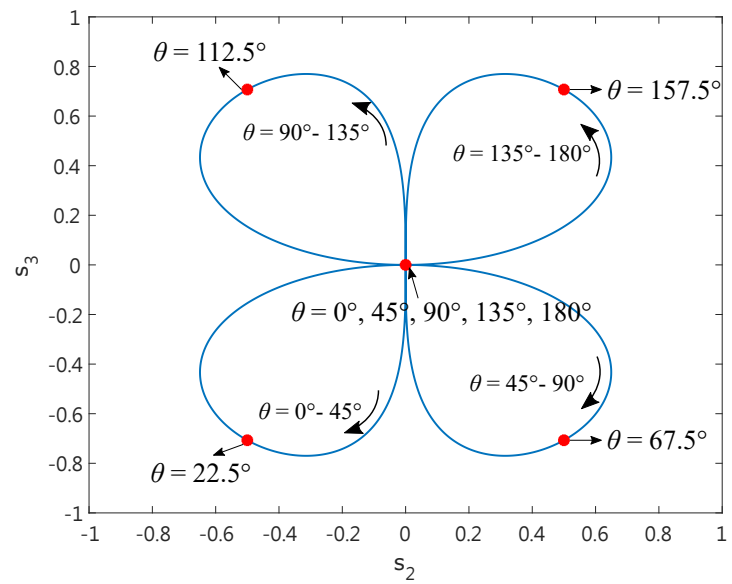

Fig. 3: Projection of the Poincaré sphere onto $s_{2}-s_{3}$ plane for the measured Stokes vector $\mathbf{S}_{\text {out }}$. deviation. Therefore, these components in the prototype should be carefully calibrated in order to obtain accurate results.

In this experiment, a Mueller-matrix ellipsometer is applied to measure Mueller matrices of all the polarization elements. The Mueller-matrix ellipsometer can be easily integrated into this prototype. The details of the Mueller matrices measurements and the calibration method of the NPBS can be found in [3]. After calibration, we obtained that the retardance of the QWP1 $\left(\delta_{1}\right)$ is $91.35^{\circ}$. The retardance $\left(\delta_{2}\right)$ and the fast axis angle $\left(\theta_{2}\right)$ of the QWP2 are $90.95^{\circ}$ and $0.04^{\circ}$. The measured Stokes vector after the NPBS and $\mathbf{M}_{\mathrm{NPBS}}^{\mathrm{r}}$ are $[1,1 .,-0.015,0 .]^{\mathrm{T}}$ and

$$
\mathbf{M}_{\mathrm{NPBS}}=\left[\begin{array}{cccc}
1 & -0.182 & 0.0042 & -0.002 \\
-0.184 & 1.004 & -0.002 & -0.003 \\
-0.002 & 0 . & 0.957 & 0.272 \\
0.003 & -0.008 & 0.282 & -0.939
\end{array}\right]
$$

From the measurement data, it is clear that the QWPs and the NPBS are not perfect elements. Hence, careful calibration of each optical element is necessary and important.

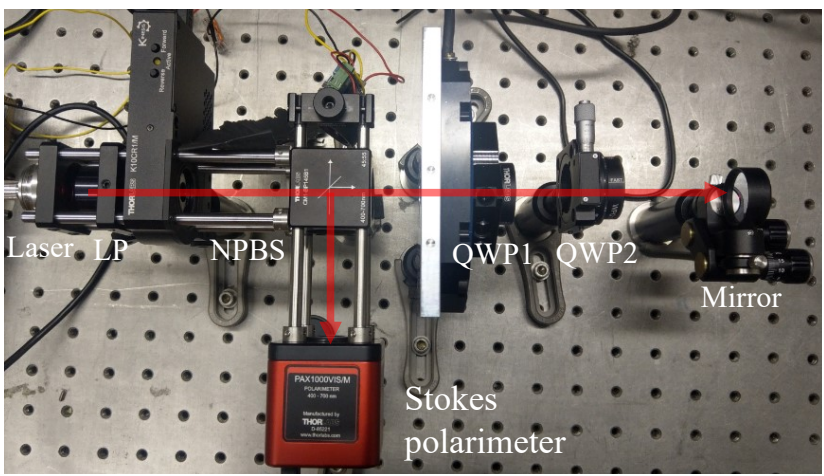

Fig. 4: Photograph of the roll-angle sensor. 


\section{Experimental result}

In Eq. 4, the fast axis angle of QWP1 can be solved by $s_{1}, s_{2}$ or $s_{3}$ analytically. Nevertheless, we used a numerical method to get higher accuracy [4]. The square error function $\chi^{2}$ can be written as

$$
\chi^{2}=\sum_{i=1}^{3}\left(s_{i}^{\text {Exp }}-s_{i}^{\text {Calc }}\left(\theta_{1}\right)\right)^{2},
$$

where the superscripts Exp and Calc indicate the experimental and calculated quantities, respectively. Non-linear optimization methods is applied to solve the fast axis angle of QWP1.

We used the piezo rotation stage as an angle reference since the stage has a high resolution $\left(0.002^{\circ}\right)$ and good repeatability $\left( \pm 0.004^{\circ}\right)$. Figure 5 shows the measurement result of the roll-angle sensor. The sensing unit (QWP1) rotates from $0^{\circ}$ to $180^{\circ}$ with a step of $20^{\circ}$. Within the whole measuring range, the measurement errors are less than $\pm 0.3^{\circ}$.

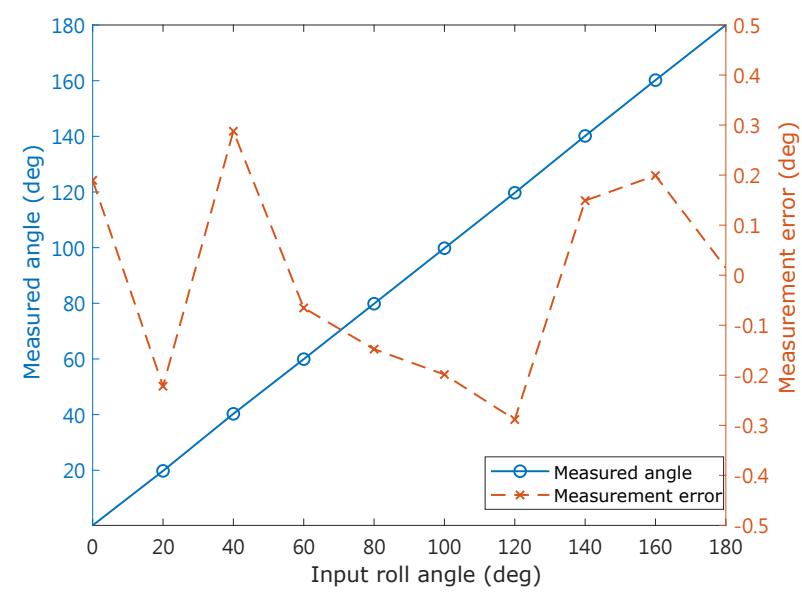

Fig. 5: Results of the measured roll angles compared with the values of the piezo rotation stage.

We also conducted a sensitivity test for the proposed roll-angle sensor. The piezo rotation stage has a minimum incremental motion of $0.002^{\circ}$. In this test, we rotated the QWP1 five times with a step of $0.006^{\circ}$. The result is shown in Fig. 6. Because the stage is in open-loop operation, the step size is not constant due to the mechanical influences, e.g., friction. It can be seen the result shows a high agreement with the rotation stage and the sensitivity of the proposed roll-angle sensor is better than $0.006^{\circ}$.

Several sources of error exist in the experiments, such as the alignment of the QWPs and the accuracy of the

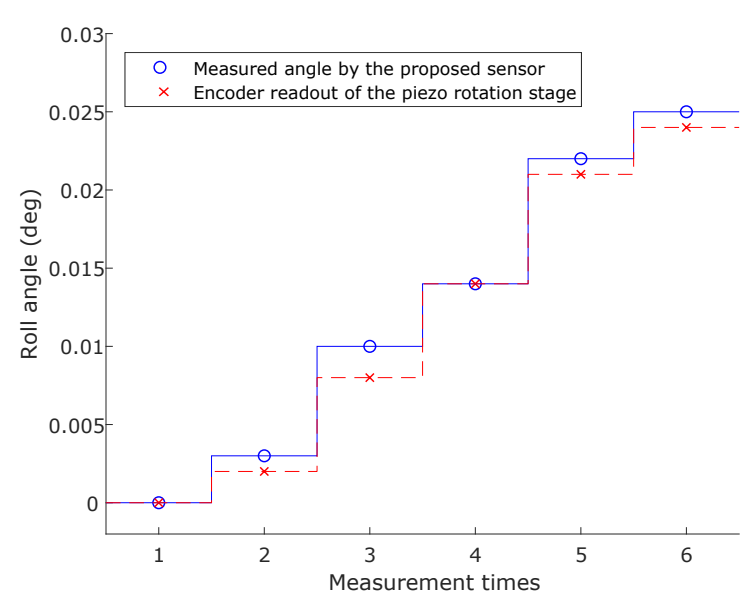

Fig. 6: Measured roll angles with steps of $0.006^{\circ}$

polarimeter. For the QWPs, the tilt angle and the incident angle cause the deviation of the retardance [8]. From the specification of the polarimeter, the azimuth and ellipticity accuracy are $\pm 0.25^{\circ}$. For the worst case, the deviation of the Stokes elements is 0.009. Moreover, the surface roughness of the mirror and the beam deviation caused by the NPBS and the QWPs might have influences on the measured Stokes parameters.

\section{Conclusion}

In this work, we proposed a novel roll-angle sensor with a resolution of $0.006^{\circ}$ and the measurement errors are less than $\pm 0.3^{\circ}$ within an unambiguous measurement range of $180^{\circ}$. The principle is based on the polarization change of the sensing unit (QWP). The polarization change can be detected by a Stokes polarimeter. The proposed sensor uses a coaxial design which decreases the complexity of optical system alignment. The measurement resolution is enhanced by using a fixed QWP and a mirror. The construction is simple and the sensor can be easily applied to existing measurement systems because of the non-contact method and the compact design. For high-speed measurements, a division-of-amplitude photopolarimeter can be used to achieve several $\mu$ s per Stokes vector $[1,19]$. In the future, we plan to evaluate the uncertainty of the roll-angle sensor and study the calibration and the stability of the system, especially for the Stokes polarimeter as the actual limiting factor of roll-angle measurements. Moreover, the sensor can be extended to 3-axis angle sensors by adding a two-dimensional position sensitive detector [17] which can 
be used for the orientation tracking. Further applications will also be studied.

\section{References}

[1] R. Azzam. Division-of-amplitude photopolarimeter (doap) for the simultaneous measurement of all four stokes parameters of light. Optica Acta: International Journal of Optics, 29(5): 685-689, 1982. ISSN 0030-3909. 10.1080/713820903.

[2] Y. Cai, B. Yang und K.-C. Fan. Robust roll angular error measurement system for precision machines. Optics express, 27(6):8027-8036, 2019.

[3] C.-W. Chen, M. Hartrumpf, T. Längle und J. Beyerer. Retroreflex ellipsometry for isotropic substrates with nonplanar surfaces. Journal of Vacuum Science \& Technology B, Nanotechnology and Microelectronics: Materials, Processing, Measurement, and Phenomena, 38(1):014005, 2020. ISSN 2166-2746. 10.1116/1.5121854

[4] X. Chen, J. Liao, H. Gu, C. Zhang, H. Jiang und S. Liu. Remote absolute roll-angle measurement in range of $180^{\circ}$ based on polarization modulation. Nanomanufacturing and Metrology, 3(3):228-235, 2020. 10.1007/s41871-020-000690 .

[5] K.-C. Fan, T.-H. Wang, S.-Y. Lin und Y.-C. Liu. Design of a dual-axis optoelectronic level for precision angle measurements. Measurement Science and Technology, 22(5):055302, 2011

[6] H. Fujiwara. Spectroscopic ellipsometry: Principles and applications. John Wiley \& Sons, Chichester, England and Hoboken, NJ, 2007. ISBN 9780470060186. 10.1002/9780470060193.

[7] S. R. Gillmer, X. Yu, C. Wang und J. D. Ellis. Robust highdynamic-range optical roll sensing. Optics Letters, 40(11): 2497-2500, 2015. ISSN 1539-4794. 10.1364/OL.40.002497.

[8] H. Gu, X. Chen, C. Zhang, H. Jiang und S. Liu. Study of the retardance of a birefringent waveplate at tilt incidence by mueller matrix ellipsometer. Journal of Optics, 20(1):015401, 2017.

[9] B. Johs und P. He. Substrate wobble compensation for in situ spectroscopic ellipsometry measurements. Journal of Vacuum Science \& Technology B, Nanotechnology and Microelectronics: Materials, Processing, Measurement, and Phenomena, 29(3):03C111, 2011. ISSN 2166-2746. $10.1116 / 1.3555332$

[10] O. Kranz, R. D. Geckeler, A. Just und M. Krause. Modelling ptb's spatial angle autocollimator calibrator. In Modeling Aspects in Optical Metrology IV, Band 8789, S. 87890D. International Society for Optics and Photonics, 2013.

[11] C. Kuang, Q. Feng, B. Zhang, Z. Zhang und S. Chen. Measurement method of the roll angle. S. 61502F. International Society for Optics and Photonics, 2006. 10.1117/12.676891. URL https://www.spiedigitallibrary.org/conferenceproceedings-of-spie/6150/61502F/Measurement-method-ofthe-roll-angle/10.1117/12.676891.full.
[12] S. Li, C. Yang, E. Zhang und G. Jin. Compact optical roll-angle sensor with large measurement range and high sensitivity. Optics Letters, 30(3):242-244, 2005. ISSN 1539-4794. 10.1364/OL.30.000242. URL https://www. osapublishing. org/viewmedia. $\mathrm{cfm}$ ? uri $=\mathrm{ol}-30-3-242 \&$ seq $=0$.

[13] Y.-C. Liu, Y.-L. Lo und C.-C. Liao. Opt. Commun., 361:153161, 2016. ISSN 00304018. 10.1016/j.optcom.2015.09.099.

[14] Z. Liu, D. Lin, H. Jiang und C. Yin. Roll angle interferometer by means of wave plates. Sensors and Actuators A: Physical, 104(2):127-131, 2003. ISSN 0924-4247. 10.1016/S09244247(03)00003-7.

[15] E. Shi, J. Guo, Z. Jia und Y. Huang. Theoretic study on new method for roll angle measurement of machines. In 2008 IEEE International Conference on Automation and Logistics, S. 2722-2726. IEEE, 92008. ISBN 978-1-4244-2502-0. 10.1109/ICAL.2008.4636635.

[16] Y. Shimizu, L. C. Chen, D. W. Kim, X. Chen, X. Li und $\mathrm{H}$. Matsukuma. An insight on optical metrology in manufacturing. Measurement Science and Technology, 2020. ISSN 0957-0233. 10.1088/1361-6501/abc578.

[17] R. Treichel, R. Sesselmann und J. Krieger. Optical sensor for measurement of roll + pitch + yaw angles over large distances with high accuracy. S. 199-207. International Society for Optics and Photonics, 1999. 10.1117/12.364285. URL https://www.spiedigitallibrary.org/conference-proceedingsof-spie/3824/0000/Optical-sensor-for-measurement-of-roll-pitch--yaw/10.1117/12.364285.full.

[18] S. Zhang, H. Gu, J. Liu, H. Jiang, X. Chen, C. Zhang und S. Liu. J. Opt-UK, 20(12):125606, 2018.

[19] S. Zhang, H. Jiang, H. Gu, X. Chen und S. Liu. High-speed mueller matrix ellipsometer with microsecond temporal resolution. Optics express, 28(8):10873-10887, 2020. 